American Journal of Pharmaceutical Education 2021; 85 (3) Article 8345.

\title{
RESEARCH
}

\section{Sterile Compounding Knowledge, Skills, and Confidence Among Graduating Doctor of Pharmacy Students}

\author{
Shandrika W. Landry, PharmD, ${ }^{\mathrm{a}}$ Brittany Singleton, PharmD, ${ }^{\mathrm{a}}$ Sara Al-Dahir, PharmD, MPH, \\ Anh Nguyen, PharmD, ${ }^{b}$ Donna S. Robinson, BS \\ ${ }^{a}$ Xavier University of Louisiana, College of Pharmacy, New Orleans, Louisiana \\ ${ }^{\mathrm{b}}$ Ochsner Medical Center, New Orleans, Louisiana \\ ${ }^{\mathrm{c}}$ Southeast Louisiana Veterans Healthcare System, New Orleans, Louisiana \\ Submitted August 14, 2020; accepted December 9, 2020; published March 2021.
}

Objective. To determine whether the exposure to sterile compounding in the pharmacy curriculum produces Doctor of Pharmacy graduates who are both competent and confident in the area of sterile compounding, and to identify additional variables that may predict student performance.

Methods. Participants were recruited from the fourth-year pharmacy class of 2018 at one university. The students were asked to complete a questionnaire assessing the following domains: demographics, confidence in compounding performance, prior experience, and theoretical knowledge. A written assessment was followed by a faculty-evaluated practicum in which the students were required to prepare two sterile products using a standardized rubric. Results were analyzed with a Student $t$ test and linear regression to determine differences in performance based upon prior experience, confidence, and theoretical knowledge.

Results. Overall, the 158 students performed well on the knowledge and skill examination, achieving an average total score of $89.8 \%$. Of the 158 total participants, the 122 survey respondents had an overall mean confidence score of 2.9 on a four-point Likert scale, with $40.2 \%$ of students scoring in the confident or very confident range of the survey. In our analysis, we found that neither prior compounding experience or self-rated confidence were predictive of students' total score.

Conclusion. The results of this study suggest that the inclusion of sterile compounding education and training in all four years of the pharmacy curriculum produces PharmD graduates who are competent, with varying levels of confidence in the area of sterile compounding.

Keywords: sterile compounding, sterile compounding assessment, compounding curriculum, student confidence, repeated exposure

\section{INTRODUCTION}

Sterile compounding is a major aspect of pharmacy practice, often performed in hospitals, health systems, and home infusion settings. Errors in sterile compounding preparation may cause irreparable harm to patients. The Institute for Safe Medication Practices (ISMP) tracks and reports such errors via the ISMP National Medication Errors Reporting Program (ISMP MERP). ${ }^{1}$ Results of the ISMP Sterile Preparation Compounding Safety Summit, released in 2013, documented several instances of sterile compounding errors. These errors related to contaminated products, incorrect drug, incorrect dose, incorrect diluent, inappropriate concentration, and product mislabeling.

Corresponding Author: Brittany Singleton, Xavier University of Louisiana, College of Pharmacy, 1 Drexel Dr., New Orleans, LA 70125. Tel: 504-520-7308. Email:

bsingle2@xula.edu
The consequences of these errors included patient harm and death. ${ }^{1}$ To improve safety and reduce errors related to sterile compounding preparations, national efforts have been made through enforceable standards, guidelines, and educational materials. At this time, regulations and guidelines on compounding sterile preparations are available from the United States Pharmacopeia (USP), the American Society of Health-Systems Pharmacists (ASHP), and the ISMP. $^{1-3}$ Despite these national efforts, errors related to sterile compounding persist. According to the 2020 State of Pharmacy Compounding Survey, 23\% of facilities surveyed over the past five years have experienced at least one patient incident involving a compounding error. ${ }^{4}$

To ensure the health and well-being of patients who receive sterile parenterals, a pharmacist must understand the theory surrounding sterile compounding, as well as develop skills of aseptic manipulation. In 2009, the ASHP and the Accreditation Council for Pharmacy Education 


\section{American Journal of Pharmaceutical Education 2021; 85 (3) Article 8345.}

(ACPE) developed a joint task force to identify entrylevel competencies required for hospital and health systems pharmacy practice, to subsequently promote their inclusion in pharmacy school curricula. ${ }^{5}$ The task force identified 25 critical practice competencies, including demonstration of aseptic technique as well as knowledge of USP Chapter 797 standards and the appropriate use of injectable medications. These specific competencies were suggested to be addressed in Doctor of Pharmacy (PharmD) curricula with introduction in didactic courses and/or practice laboratories and reinforcement in introductory and advanced pharmacy practice experiences (IPPEs and APPEs). The recommended methods of competency assessment were examination and direct observation by preceptors. ${ }^{5}$

In 2010 the American Association of Colleges of Pharmacy (AACP) surveyed its pharmaceutics and pharmacy practice section members to assess the status of compounding education within their institutions and determine an appropriate compounding curriculum for basic and advanced compounding. The 137 faculty respondents agreed on the importance of sterile compounding courses within the pharmacy school curriculum and recommended that three to four credit hours per curriculum be devoted to compounding education. Similar to ASHP/ ACPE recommendations, AACP also supported required laboratory training for students and assessment by direct observation. ${ }^{6}$ A collaborative 10 -year comparison study was conducted by Dana and colleagues ${ }^{7}$ and Cooper and colleagues $^{8}$ to describe instruction on sterile compounding within US pharmacy schools using data from 2007 and 2017. Findings showed a significant increase in technique observation, hands-on laboratory-based training, and compounding assessment. Despite these positive trends, few respondents expressed confidence in students' preparation for experiential education and practice. ${ }^{7,8}$

Although curriculums have increased sterile compounding content, adequate reinforcement is necessary for compounding skill mastery and long-term skill retention. ${ }^{9,10}$ Providing students with repeated opportunities to refine their skills before having them engage in real-world practice has resulted in improvement in aseptic technique, fewer compounding errors, and increased student confidence. ${ }^{11-14}$ A study by Kosinski and colleagues demonstrated that with repeated exposure to compounding techniques within a laboratory course, greater than $90 \%$ of students' sterile compounding skills were retained 18 months later. ${ }^{11}$ In contrast, Eley and colleagues' study of pharmacy students' retention of compounding skills showed that, without any repeated exposure to previously learned skills, a majority of students had not maintained competency when assessed one year later. ${ }^{15}$ This evidence highlights the need for providing multiple curricular opportunities to practice compounding skills, which will enhance students' confidence and help ensure their practice readiness.

For these reasons, sterile compounding is covered repeatedly within the Xavier University of Louisiana College of Pharmacy curriculum, utilizing both didactic and hands-on methods (Figure 1). Students begin the sterile compounding instruction in the spring semester of their first year. Early, didactic, and hands-on experience is gained in the Pharmaceutics I laboratory course. The Pharmaceutics II lecture course builds on principle compounding concepts, and practical skills are reinforced via simulated and directly applied, institutional-based compounding experiences in the Introductory Pharmacy Practice Experience II course. Content is expounded on didactically in the Pharmacy Practice lecture and Pharmacy Practice laboratory courses, which include handson practice and assessment. Within the final year of the curriculum, the Professional Experience Program (PEP) Meeting course is a required co-requisite for all APPE students in which final capstone assessments are administered. This course contains a sterile compounding didactic reinforcement session and an objective structured clinical examination (OSCE). During the capstone compounding OSCE, competence was assessed by both written examination and direct observation of compounding skills by trained faculty members. The primary goal of this study was to determine whether repeated exposure to sterile compounding in the pharmacy curriculum produces pharmacy graduates who are both competent and confident in the area of sterile compounding. A secondary goal was to identify additional variables that may predict student performance.

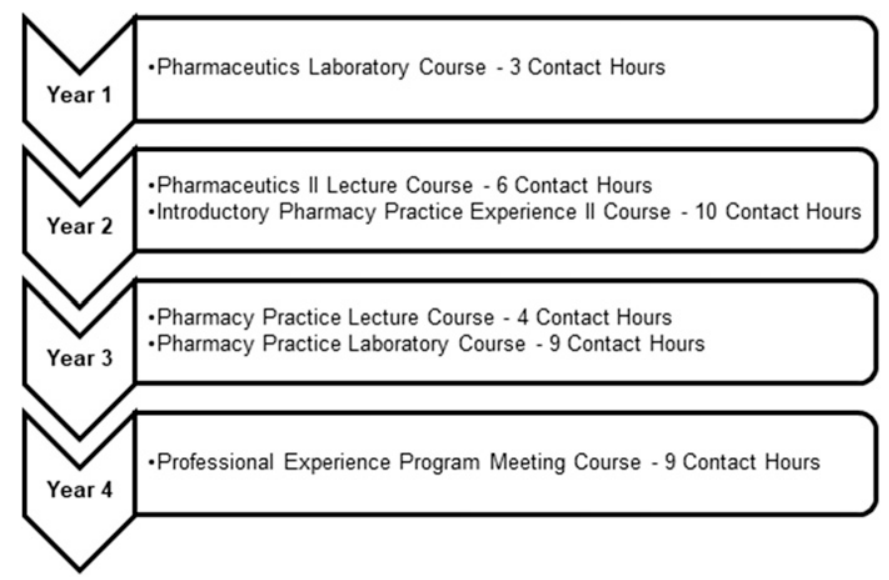

Figure 1. Sterile Compounding Exposure Within the College of Pharmacy Curriculum 


\section{American Journal of Pharmaceutical Education 2021; 85 (3) Article 8345.}

\section{METHODS}

This research was approved by the Institutional Review Board at Xavier University of Louisiana. During the 2017-2018 academic year, fourth professional year PharmD students were scheduled to complete the sterile compounding capstone assessment during the fall semester or spring semester. Prior to completing the sterile compounding assessment, all students participated in a mandatory examination preparation session, during which a faculty member reviewed the scoring rubric with students and outlined the examination procedures for them.

During this session, students completed an optional survey to examine factors that might impact their performance on the skills and/or knowledge assessment. The nine-item survey examined exposure to sterile compounding training outside of the curriculum and within the curriculum via their practice experiences. The survey also assessed students' sterile compounding confidence and perceived need for additional training. Students used a four-point Likert scale $(1=$ not confident, 2=somewhat confident, $3=$ confident, $4=$ very confident) to respond to survey items. Finally, students were asked to indicate their intended future practice setting. After participating in the examination preparation session and having an opportunity to practice their hands-on compounding skills, students completed the capstone examination.

The examination was twofold, consisting of both a knowledge and skills assessment. The knowledge assessment contained four multiple-choice questions that examined the students' knowledge of the following key areas related to sterile compounding: proper gowning/ garbing, maintaining sterility, antimicrobial risk levels, and primary engineering controls. There was also one open-entry question that required the student to perform common calculations involved in preparing compounded sterile preparations. During each administration of the knowledge assessment, 16 different versions were used to minimize the likelihood that sharing of exam information between students would impact exam performance.

The skills assessment required the students to demonstrate their ability to accurately perform the following two tasks using appropriate aseptic technique. Task 1: Dissolve a simulated drug powder for reconstitution with $5 \mathrm{~mL}$ of $0.9 \%$ sodium chloride from a large volume intravenous (IV) fluid bag and then remove $5 \mathrm{~mL}$ of the dissolved product and inject into a $50 \mathrm{~mL} 0.9 \%$ sodium chloride IV bag. Task 2: Open and remove $2 \mathrm{~mL}$ of a simulated drug solution from a glass ampule and inject the solution into a $50 \mathrm{~mL} 0.9 \%$ sodium chloride IV bag. Each student was observed and their performance was evaluated by a trained faculty member using a standardized scoring rubric. The scoring rubric that was developed was prescriptive and included each step in the sterile compounding process that the students were expected to perform (Figure 2).

To determine predictors of student performance, comparisons were made between each student's total score and their prior compounding experience, intention to pursue employment in a setting where the instructors deemed that compounding skills would be required, and their self-rated confidence in the knowledge domains covered by the assessment, as well as their confidence in their hands-on compounding skills. Further comparisons were made between students' overall knowledge performance and their compounding skill performance. Additionally, overall skill performance was compared

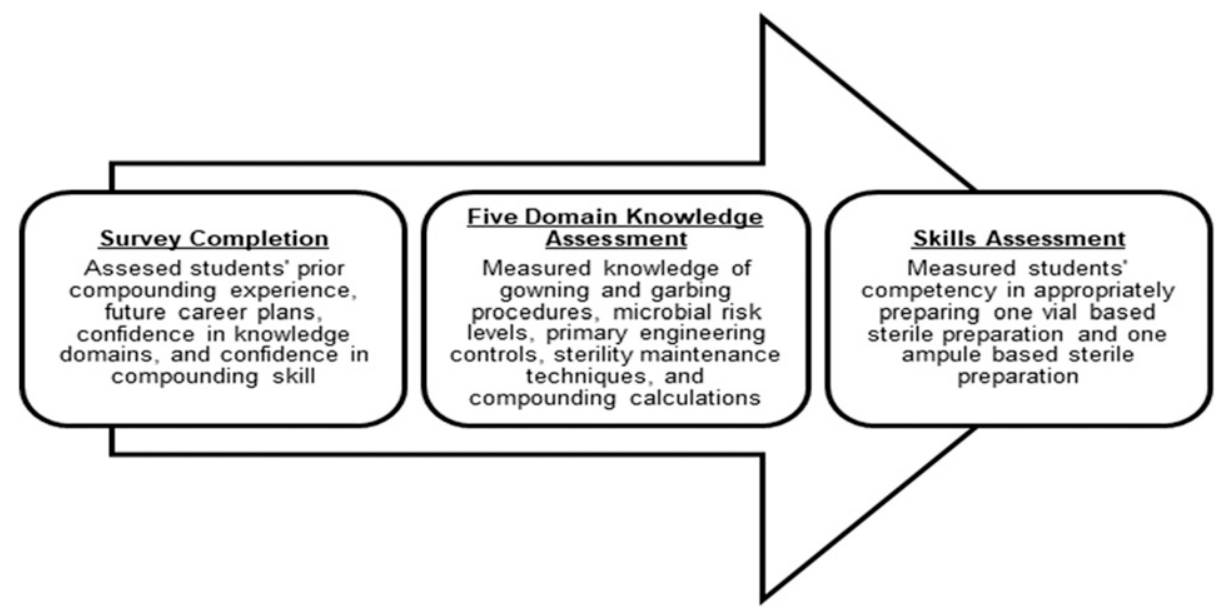

Figure 2. Survey and Assessment Procedure Diagram 


\section{American Journal of Pharmaceutical Education 2021; 85 (3) Article 8345.}

between vial-based product preparation and ampulebased product preparation.

A paired $t$ test was used to compare performance between the written and practicum components of the assessment. Furthermore, the paired $t$ test was used to compare performance among subcomponents of the skills assessment, such as vial vs ampule preparation. A bivariate linear regression was used to determine individual predictors of performance based upon demographic variables, previous experience in sterile compounding, and overall confidence in the sterile compounding technique. Finally, a multivariable linear regression comparing Akaike information criteria (AIC) was performed to determine the best fit model for final predictors associated with improved performance on the composite score, theoretical and practicum based, for sterile compounding. All analysis was conducted with Stata/IC, version 15 (StataCorp).

\section{RESULTS}

Of the 158 students who completed the assessment, 122 also completed the survey (response rate, 77\%), which included questions on prior experience and confidence. The average age of survey respondents was 26.9 years. Most (66\%) were female. Nearly one-third of students $(30 \%)$ indicated they had prior extracurricular sterile compounding experience, and 35\% indicated that they intended to work in a setting where sterile compounding would be commonplace.

Overall students performed well on the assessment, with an average total score of $89.8 \%(\mathrm{SD}=7.3 \%)$. Students performed better on the skills portion of the assessment than on the written portion. Students performed slightly better when compounding an ampule-based preparation than they did when compounding a vial-based preparation (Table 1). Of the five domains covered in the written assessment,

Table 1. Performance of Students Who Received Instruction in Sterile Compounding Throughout the Four-Year Curriculum of a Doctor of Pharmacy Program, $\mathrm{N}=158$

\begin{tabular}{lcc}
\hline & Mean Score (SD) & $\boldsymbol{p}$ value \\
\hline Total Composite Score & $89.8(7.3)$ & \\
Student performance on written vs skill assessments & \\
Written assessment & $83.2(18.3)$ & $<.001^{\mathrm{a}}$ \\
Overall skills assessment & $90.8(8.0)$ & \\
Student performance on vial vs ampule-based skill & \\
assessments & & \\
Vial preparation & $89.9(9.6)$ & $.006^{\mathrm{a}}$ \\
Ampule preparation & $92.0(8.5)$ & \\
\hline
\end{tabular}

${ }^{a}$ Paired t-tests were used to compare performance between the components of the assessment. Significance was defined as $p<.05$ fewer than $80 \%$ of students correctly answered the questions regarding sterility maintenance techniques (74\%) and antimicrobial risk levels (77\%), though this is a formative assessment of previously completed content in the first, second, and third years. For the skills assessment, fewer than $80 \%$ of students successfully avoided touch contamination of critical sites during the manipulation process, or appropriately used a filter needle when compounding an ampule-based preparation (78\%).

Survey respondents had an overall mean confidence score of $2.9(\mathrm{SD}=0.5)$ on a four-point Likert scale, with $40.2 \%$ of students scoring in the confident (3) or very confident (4) range of the survey. Students expressed the least confidence in primary engineering controls $(\mathrm{M}=2.2$, $\mathrm{SD}=0.9)$ and antimicrobial risk levels $(\mathrm{M}=2.8, \mathrm{SD}=0.9)$. Students expressed the greatest confidence in apparel and proper gowning technique $(\mathrm{M}=3.4, \mathrm{SD}=0.5)$ and common calculations related to sterile compounding $(\mathrm{M}=3.3$, $\mathrm{SD}=0.6$ ). Finally, students expressed equal levels of confidence based on mean scores of the Likert scale in compounding both vial and ampule-based preparations $(\mathrm{M}=3.3, \mathrm{SD}=0.7$ vs $\mathrm{M}=3.2, \mathrm{SD}=0.7)$. Students with prior extracurricular compounding experience had higher confidence scores compared to those who did not. Those who intended to pursue careers in which compounding was relevant did not have significantly higher confidence scores than those who did not (Table 2).

In the bivariate regression, neither gender at birth, age, prior compounding experience, intention to pursue a compounding career, nor overall confidence in compounding were individual predictors of overall total composite score. Students with extracurricular compounding experience had slightly lower scores than those without, though this difference did not reach significance. Those who intended to practice in a sterile compounding setting also had a nonsignificant lower total score than those who did not intend a sterile compounding setting for future practice (Table 2). Furthermore, in the multivariable analysis, none of the demographic variables such as gender, age, and work experience served as significant predictors for improved composite score performance.

\section{DISCUSSION}

Based on our findings, the repeated exposure of students to sterile compounding in this college of pharmacy curriculum produced graduates who are competent in the area of sterile compounding. Confidence in sterile compounding varied across students based on previous work experience. This was evidenced by the average total assessment score of $89.8 \%$ and a mean total confidence score of 2.9 on a four-point Likert scale. These results are encouraging considering that the curriculum at the Xavier 


\section{American Journal of Pharmaceutical Education 2021; 85 (3) Article 8345.}

Table 2. Mean Total Performance and Confidence Score Comparisons Among Fourth-Year Doctor of Pharmacy Students Based on Prior Relevant Pharmacy Practice or Intended Future Relevant Practice

\begin{tabular}{lccc}
\hline & Yes & No & $p$ value \\
\hline Prior Relevant Pharmacy Practice - Difference in Average & Confidence and Average Total Score & \\
Prior relevant experience & $(\mathrm{n}=36)$ & $(\mathrm{n}=86)$ & $<.05^{\mathrm{a}}$ \\
Confidence, Likert scale 1-4, Mean (SD) & $3.0(0.6)$ & $2.8(0.5)$ & .50 \\
Mean total composite score (\%) (SD) & $89.1(6.9)$ & $90.1(7.4)$ & \\
Intended Future Relevant Practice - Difference in Average Confidence and Average Total Score & $(\mathrm{n}=79)$ & .06 \\
Future relevant practice & $(\mathrm{n}=43)$ & $2.8(0.5)$ & .14 \\
Confidence, Likert scale 1-4, Mean (SD) & $3.0(0.6)$ & $90.5(6.9)$ & \\
Mean total composite score (\%) (SD) & $88.5(7.8)$ &
\end{tabular}

${ }^{a}$ Bivariate linear regression was used to determine individual predictors of performance. Significance was defined as $p<.05$

University of Louisiana College of Pharmacy embodies several recommendations from AACP and ASHP regarding the design of curricular content related to sterile compounding. 5,6

Students demonstrated significantly stronger performance on the skills assessment than on the written assessment. This may be explained by examination design. Although students were given both written and skills assessments within the curriculum, the skills portion typically comprises a higher percentage of assessment grades. Therefore, students may place a greater emphasis on hands-on skill preparation, because that particular section is most critical to their overall examination performance. Our faculty support this examination design to ensure student acquisition of critical aseptic manipulation skills. However, based on the results of our analysis, additional emphasis on sterile compounding theory may be warranted, particularly regarding the topics of sterility maintenance techniques and antimicrobial risk levels, which were the two domain areas on the written assessment in which students performed the worst.

Skills scores were slightly higher for ampule manipulation than for vial manipulation. This may be due in part to the vial manipulation being a more complex process, requiring additional steps and creating more opportunity for first air blockage and other errors in aseptic technique. More than twice as many students performed touch contamination errors during vial manipulation than during ampule manipulation. When examining the skill competency areas with less than $80 \%$ appropriate performance, we recognize an indication for increased student training regarding the avoidance of touch contamination, particularly concerning vial manipulations. Additionally, the appropriate use of a filter needle when compounding an ampule-based preparation must be reinforced.

Prior compounding experience was positively associated with student confidence but was not a predictor of skill level. Neither prior extracurricular sterile compounding exposure, future intention to practice sterile compounding, or self-rated confidence in knowledge and skills resulted in better assessment performance. The fact that none of the variables examined were predictors of students' total score suggests that student performance was largely dependent upon their exposure to sterile compounding within the curriculum. This highlights the importance of ensuring that students are provided enough opportunities to practice these skills when designing a curriculum.

In our investigation of existing literature examining sterile compounding at other colleges of pharmacy, we identified several recent, relevant publications. Our results reinforce the findings of previous studies, which promote repeated exposures to sterile compounding and the inclusion of both hands on and didactic training. ${ }^{11-13}$ Our research study differed from the recently published literature in several ways. One major difference was that we examined graduating students' sterile compounding knowledge and abilities after exposure to compounding throughout the entire PharmD curriculum. The studies by Diehl and colleagues and Monestime and colleagues focused primarily on the redesign of a sterile compounding course and examined student performance after the completion of a single course on compounding. ${ }^{16,17}$ Additionally, other studies did not examine external factors as possible predictors of students' compounding performance.

This study had several limitations. The first of these was the sample size, which included only one graduating class cohort from one institution. This limited measure, combined with our survey response rate of $77 \%$, lessens the reliability and external validity of our results. Another limitation was that our skills assessment only consisted of compounding vial- and ampule-based sterile preparations. Other critical tasks such as aseptic handwashing, garbing and gowning, gloved fingertip sampling, primary engineering control cleansing and maintenance, and end- 


\section{American Journal of Pharmaceutical Education 2021; 85 (3) Article 8345.}

product testing were not performed or assessed. This was because of several factors, including assessment time constraints, the availability of trained faculty and staff members, and financial constraints.

An additional limitation of our assessment included the format of the written knowledge assessment. For the purposes of time management, the knowledge assessment only consisted of one question per knowledge domain, comprising five questions. In an effort to maintain the integrity of the knowledge assessment, 16 different versions were used. Because there were multiple versions of the knowledge assessment with only one question asked per domain, students had a limited ability to demonstrate competency in each domain. Had a student received an alternate version of the knowledge assessment, they may have been able to correctly answer a different question in that same domain.

In addition to providing students with study materials and access to demonstration videos that guided them through each step of the physical compounding process, students were also given the opportunity to practice their compounding skills in the week before the assessment occurred. Some students did not take advantage of this opportunity, which could have negatively impacted their performance.

Based on best practices established in the literature and the research findings and the curricular design described here, we suggest introducing sterile compounding within the first year of the PharmD curriculum and reinforcing knowledge and hands-on skills in each subsequent year of the curriculum within both didactic and experiential courses. Additional considerations when determining curricular coverage of sterile compounding may be to incorporate quality assurance measures, such as gloved fingertip sampling to objectively assess students' handwashing, garbing and gowning skills, and end product sterility or potency testing to assess student accuracy and aseptic technique. ${ }^{5,13,14,18,19}$ Inclusion of immersive simulation techniques that emulate real world scenarios and practical challenges may serve to develop students' problem-solving abilities, enhance skill retention, promote practice readiness, and ultimately ensure patient safety. ${ }^{16,17}$

\section{CONCLUSION}

The results of this study indicate that ongoing exposure of pharmacy students to sterile compounding in all four years of a PharmD curriculum produces graduates who are competent in this vital area of pharmacy practice. Students' confidence in sterile compounding varied based on their previous compounding experience. Upon analysis, the external factors of prior compounding experience and self-rated confidence were not predictive of student performance on the assessment. Increased curricular emphasis may be warranted, particularly regarding the topics of sterility maintenance techniques, antimicrobial risk levels, avoidance of touch contamination, and the appropriate use of filtration when working with ampules. Future studies may focus on the curricular impact of sterile compounding training in multiple cohorts and institutions.

\section{ACKNOWLEDGMENTS}

The authors thank Dr. Christopher Williams of Xavier University of Louisiana for his review of this manuscript.

\section{REFERENCES}

1. Rich DS, Fricker MP, Cohen MR, Levine SR. Guidelines for the safe preparation of sterile compounds: Results of the ISMP sterile preparation compounding safety summit of October 2011. Hosp Pharm. 2013;48(4):282-294, 301. doi:10.1310/hpj4804-282

2. ASHP Guidelines on compounding sterile preparations. Am J Health Syst Pharm. 2014;71(2):145-166. doi:10.2146/sp140001 3. USP General Chapter Pharmaceutical Compounding-Sterile Preparations. United States Pharmacopeia and National Formulary (USP42-NF37): Rockville, MD; United States Pharmacopeial Convention; 2008:1-43.

4. State of Pharmacy Compounding 2020. Pharmacy Purchasing \& Products. 2020;17(4):1-60.

5. American Society of Health-System Pharmacists. Entry-level competencies needed for pharmacy practice in hospitals and healthsystems. https://www.ashp.org/-/media/assets/pharmacy-practice/ resource-centers/inpatient-care/entry-level-competencies-neededfor-pharmacy-practice-in-hospitals-and-health-systems.ashx. Accessed August 1, 2020.

6. Shrewsbury R, Augustine S, Birnie C, Nagel K. Assessment and recommendations of compounding education in AACP member institutions. Am J Pharm Educ. 2012;76(7). doi:10.5688/ajpe767s9 7. Dana KN, Hertig JB, Scott CR, Briscoe-Dwyer L. Instruction on compounded sterile preparations at schools of pharmacy. Am J Health Syst Pharm. 2018;75(12):847-849. doi:10.2146/ajhp170636

8. Cooper C, Almgren MM, Maxwell WD, Baker JL. Instruction on compounded sterile preparations at schools of pharmacy. Am J Health Syst Pharm. 2018;75(12):845-847. doi:10.2146/ajhp170641 9. Karpicke J, Roediger H. J Mem Lang. 2007;57(2):151-162. doi: 10.1016/j.jml.2006.09.004

10. Mudit M, Alfonso LF. Analytical evaluation of the accuracy and retention of compounding skills among PharmD students. Am J

Pharm Educ. 2017;81(4):64. doi:10.5688/ajpe81464

11. Kosinski TM, Brown MC, Valdovinos K, Zavala PJ. Acquisition and retention of sterile compounding accuracy skills. Am J Pharm Educ. 2017;81(6):115. doi:10.5688/ajpe816115

12. Cretton-Scott E, Cruthirds D, Coward L. Incorporation of handson sterile technique instruction in an introductory pharmacy practice experience. Am J Pharm Educ. 2015;79(2):28. doi:10.5688/

ajpe 79228

13. Isanhart CM, Mccall KL, Kretschmer D, Grimes BA. Parenterals laboratory course to reduce microbial contamination rates in media fill tests performed by pharmacy students. Am J Pharm Educ. 2008; 72(2):27. doi:10.5688/aj720227 


\section{American Journal of Pharmaceutical Education 2021; 85 (3) Article 8345.}

14. McGill JE, Holly DR. Integration of pharmacy practice and pharmaceutical analysis; quality assessment of laboratory performance. Am J Pharm Educ.1996;60(Winter):370-374.

15. Eley JG, Birnie C. Retention of compounding skills among pharmacy students. Am J Pharm Educ. 2006;70(6):132. doi:10.5688/ aj7006132

16. Diehl B, Dy-Boarman EA, Bottenberg MM, Mobley-Bukstein W. Utilizing desirable difficulties for sterile compounding training in a skills-based laboratory course. Curr Pharm Teach Learn. 2018; 10(4):469-472. doi:10.1016/j.cptl.2017.12.018
17. Monestime S, Thomas D, Hooper CD, Day T, Suzuki S, Martin $\mathrm{RD}$. Instructional and assessment redesign of a sterile compounding course using immersive simulation. Am J Pharm Educ. 2019;84(2): 7473. doi:10.5688/ajpe 7473

18. Nemec EC, Petraglia C, Mattison MJ. Design considerations of a compounded sterile preparations course. Am J Pharm Educ. 2016; 80(1):12. doi:10.5688/ajpe80112

19. Lee RK, Shrewsbury RP. Potency analyses provide insight into student aseptic compounding technique errors. Am J Pharm Educ. 2019;83(9):7338. doi:10.5688/ajpe7338 